\title{
Extra-corporeal Membrane Oxygenation Procedures on YouTube: What Practices Have Being Show
}

\author{
(1) Tuğra Gençpınar1, (1) Çağatay Bilen², (i) Serdar Bayrak1, (i) Gökmen Akkaya2, (1) Volkan Hancı, \\ (1) Sibel Büyükçoban3
}

1Dokuz Eylül University Faculty of Medicine, Department of Cardiovascular Surgery, İzmir, Turkey

${ }^{2}$ Ege University Faculty of Medicine, Department of Cardiovascular Surgery, İzmir, Turkey

${ }^{3}$ Dokuz Eylül University Faculty of Medicine, Department of Anesthesiology and Reanimation, İzmir, Turkey

\begin{abstract}
Objectives: To the our best knowledge, there is no literature on the effectiveness of YouTube on extracorporeal membrane oxygenation (ECMO) practice. In our study, we aimed to evaluate the characteristics and medical aspects of videos on YouTube about ECMO.

Materials and Methods: In internet media website YouTube.com search engine; the word ECMO was searched in December 2018 without any filter. The listed first 100 videos were classified according to count of like, dislike, origin of country and types of ECMO. ECMO videos were categorized under three main topics as, educational purpose, patient follow-up and weaning from ECMO. The materials were evaluated in terms of intelligibility using the suitability assessment of materials (SAM). User participation measurements were obtained for each video.

videos, content variety and duration of ECMO contents that were uploaded to YouTube after 2016 increased significantly. However, it was found that there was no significant relationship between date and duration of SAM and there was no significant correlation between the duration of the video and the SAM score. However, it was determined that there was a positively weak correlation between the scores of the videos and the number of views.

Conclusion: Medical education is forming from didactic education to a system which is problem-based, media and visually aided, and effectively using all of the educational tools. During this period, the use of sites such as YouTube is increasing among medical education sources. Considering that, the discussions may be based on insufficient information quality, patient confidentiality and legal issues.

Results: In our study, it was observed that the number of Thus, we think that the necessity of double-blind peer
\end{abstract}

\footnotetext{
Address for Correspondence: Tuğra Gençpınar, Dokuz Eylül University Faculty of Medicine, Department of Cardiovascular Surgery, İzmir, Turkey

e-mail: tugra01@yahoo.com ORCID ID: orcid.org/0000-0003-4438-7991

Received: 06.03.2019 Accepted: 13.03.2019
}

Cite this article as: Gençpınar T, Bilen Ç, Bayrak S, Akkaya G, Hancı V, Büyükçoban S. Extra-corporeal Membrane Oxygenation Procedures on YouTube: What Practices Have Being Show. EJCM 2019;7(1):28-35.

DOI: 10.32596/ejcm.galenos.2019.00013

${ }^{\circ}$ Copyright 2019 by Heart and Health Foundation of Turkey (TÜSAV) / E Journal of Cardiovascular Medicine published by Galenos Publishing House. 
reviews is one of the important points to discuss in terms of content, educational direction, patient confidentiality and legal procedures in social media related to medical education.
Keywords: Medical knowledge, e-learning, YouTube, femoral arter, ECMO, cardiovascular surgery, web-based learning

\section{Introduction}

With the development of the internet, as in all other disciplines, the speed of information exchange and the use of communication media have increased considerably in the health services sector. In the active medical education system, students are encouraged to have access to current online publications and visual aids ${ }^{(1)}$. YouTube video sharing is one of the most frequently used one in social media for educational purpose. Raikos and Waidyasekara found that shared 294 videos were involving beneficial scientific data for medical students on the YouTube about the surgical anatomy of the heart ${ }^{(2)}$. The researchers stated that these can contribute to clinical branches by sharing more.

Medical educators, students, practitioners, physicians, auxiliary health personnel, and even patients often follow YouTube's relevant visual documents and videos on the website in order to visually learn and interpret medical conditions. YouTube is a video hosting website, locates in San Bruno, California, United States of America ${ }^{(1,2)}$. Approximately, 100 videos have being added by users worldwide in every minute ${ }^{(1)}$. However, these videos cannot be verified and are submitted by non-peer-reviewed sources. As a result, such studies analyzing YouTube's educational content have been reporting limited results. Moreover, these studies were not able to exclude noneducational videos from unreliable sources. On the other hand, several articles declare that YouTube videos which have been prepared with reliable sources and suitable for educating the public have a high educational value. Reliable videos on cardiovascular diseases are watched in terms of compliance with treatment, screening, or prevention ${ }^{(1-6)}$. Since 2007, various results on YouTube's educational content have been reported ${ }^{(1-9)}$. As a consequence of these varied findings, researches involving medical YouTube videos decreased by $83 \%$ in one year ${ }^{(9-1)}$. Desai et al. have evaluated 139 YouTube videos for global laparoscopic techniques for cholecystectomy, therefore, suggested some YouTube videos for surgical techniques ${ }^{(11)}$. Furthermore, thereby considering the Spearman correlation coefficient (SCC) for the development of surgical applications, they have concluded that the technological infrastructure provides convenience in surgical practice. The value of SCCs is feasible in grading and assessing about visual video quality and utility.

Extra-corporeal membrane oxygenation (ECMO) is one of the most important and widely used medical device that provides lung or heart circulation support in reversible conditions of cardiac or respiratory failure, therewithal mostly utilized in case of failure to weaning from the cardiopulmonary by-pass $(\mathrm{CPB})^{(11-13)}$. ECMO may be implanted in order to bridging for long-term ventricular assist device in cardiomyopathies or in case of cardiac arrest due to any reason, and patients where the patient is unstable or unresponsive to the cardiopulmonary resuscitation (CPR). Nonetheless, in high-risk catheter interventions, the ECMO can be fitted as an elective support either. However, the issue of which type of ECMO should be installed is also important. For instance, in case of ischemic cardiogenic shock or when the patient failed to wean from CPB after cardiac surgery, venoarterial ECMO support is preferred. On the other hand, veno-venous ECMO is frequently chosen in patients with reversible lung pathologies.

Recent articles regarding ECMO's indications, followup, complications, positive contribution to transplantation are frequently studied in the literature. The aim of this 
study is to review the ECMO videos in YouTube and to discuss them with literature.

\section{Materials and Methods}

Between the dates of 1-30 December 2018, by searching the keyword "ECMO" in YouTube search, we have determined the videos with medical content. Then we listed the first 100 videos focused on the cardiac, vascular or cardiovascular fields. The number of likesdislikes, country origins of the videos which were related to ECMO treatment had been investigated. In terms of the number of views, likes, and comments, the evaluation of intelligibility was performed by using the suitability assessment of materials (SAM) by examining the videos for medical education ${ }^{(11)}$. User participation measurements were reviewed for each video. Most of the videos were involving the subjects of ECMO's functioning, implementation or had a feature of being educational videos. In assessment, researchers focused on the type, duration and history of ECMO video content. Due to there is no verified scoring system available for the videos yet; the video contents was categorized according to 7 different educational contents as; 1) ECMO initiation, 2) ECMO monitoring, 3) ECMO termination, 4) ECMO indications, 5) ECMO complications, 6) treatment/management and, 7) preventing ECMO complications. Our study has also adopted this quantitative method in data collection and analysis. Moreover, we developed a research method basing on behavioral appreciation and follow-up rates ${ }^{(11)}$.

Our study is based on a web research therefore ethical approval did not obtained and does not include any patient, for this reason there was no need to obtain an informed consent form.

\section{Evaluating the Eligibility of Videos}

SAM evaluated the videos by using variables as; 1) content of the videos, 2) level of informativeness, 3) graphics, 4) order and typography, 5) learning stimulation and, 6) cultural fitness variables then a separate SAM score was calculated for each video ${ }^{(11)}$.
Following a rating of 100 over SAM, videos with 0-39 cumulative raw score was identified as "insufficient", 40-69 cumulative raw score was accepted as "sufficient" and 70100 cumulative raw scores were considered as "superior".

\section{Evaluating the User Engagement}

Five user interaction measurements recorded for each video; 1) views, 2) likes, 3) dislikes, 4) favorites and, 5) comments. These data were collected between the dates of 1-30 December 2018.

\section{Statistical Analysis}

Acquired data was analyzed using a SPSS (Statistical Package for Social Sciences, Chicago, IL, USA) 20.0 software. Kruskal-Wallis and Mann-Whitney U tests were used to compare the groups. A $p$ value less than 0.05 was considered as significant difference.

\section{Results}

In our study, the listed first 100 videos following a search with ECMO keyword in the YouTube search engine between the dates of 1-30 December 2018 were evaluated. Overall 13 hours 20 minutes and 38 seconds of footage were examined. The longest video was 1 hour 54 minutes and 46 seconds, meanwhile the shortest one was 28 seconds. The most liked video had 223 likes while the least one had none. Nonetheless, the most viewed video was viewed for 117.955 times, this number was minimum 32 for the least. The number of comments for videos was varying between 0 and 223 . The average number of views was $14956.14 \pm 22044.00$, while the average number of "likes" was $33.33 \pm 48.29$; the mean number of "dislike" was found to be $2.44 \pm 4.05$ and the mean video duration was observed as $13.11 \pm 17.32$ minutes.

Twelve (12\%) of the videos were containing animation. Seventeen $(17 \%)$ of the videos provide information on cannulation and $8(8 \%)$ of them on decannulation. There withal, 37 (37\%) of the videos were about ECMO indications, 36 (36\%) were about ECMO complications. On the other hand, 33 (33\%) of all videos were mentioning treatment and management of complications, and 31 (31\%) of them were related to prevention of complications. 
Eighteen (18\%) of the videos were containing a footage from operating room. Fifty six (56\%) videos were containing information about veno-arterial ECMO, while 48 (48\%) video were involving information about veno-venous ECMO. While 61 (61\%) of the videos were for educational purposes, $56(56 \%)$ videos were simply explaining the operation principle of ECMO. Twenty three (23\%) videos were including radiological examinations that used during cannulation, follow-up or complications. The videos were evaluated according to SAM score. The highest score was $36(95 \%)$ and the lowest one was calculated as $3(8 \%)$. When the videos had evaluated via SAM score, 21 videos were in the insufficient group and 49 were in sufficient group. Besides, 30 videos were found in superior group.

There was no significant correlation between the duration of the video and the SAM score $(p>0.05)$. A positive correlation was found between the SAM score of the videos and the number of views $(\mathrm{p}=0.046 ; \mathrm{r}=0.200)$. Similarly, there was a positive correlation between the percentage of SAM and the number of views ( $p=0.042$; $\mathrm{r}=0.204$ ). However, there was no detected significant correlation between SAM score and number of likes, dislike, and comments. There was a positive correlation between the number of views and number of likes $(\mathrm{p}<0.001 ; \mathrm{r}=0.689)$, dislikes $(\mathrm{p}<0.001 ; \mathrm{r}=0.663)$ and comments $(\mathrm{p}<0.001 ; \mathrm{r}=0.645)$. Moreover, no significant difference was found between the SAM groups and the origin of countries $(\mathrm{p}=0.725)$.
When the videos are reviewed by uploading years, $4 \%$ was loaded into YouTube before 2010, while 36\% were between years 2011-2015 and 60\% were after 2016. This finding may be interpreted as that the use of YouTube as a source of information concerning ECMO is increasing recently (Table 1: video content distribution) ${ }^{(1-3)}$. According to SAM groups and within advancing years, video contents and characteristics demonstrated statistically significant changes (Table 2: video characteristics according to years and SAM groups), [Table 3: comparison of the content of

Table 1. Video content distribution

\begin{tabular}{|l|l|l|l|}
\hline Video content & $\mathbf{+ ( \% )}$ & $\mathbf{- ( \% )}$ & Total (\%) \\
\hline Animation & 12 & 88 & 100 \\
\hline Cannula procedure & 17 & 83 & 100 \\
\hline ECMO monitoring & 32 & 68 & 100 \\
\hline Decannulation & 8 & 92 & 100 \\
\hline Indication & 37 & 63 & 100 \\
\hline Complication & 36 & 64 & 100 \\
\hline Treatment management & 33 & 67 & 100 \\
\hline Complication prevention & 31 & 69 & 100 \\
\hline Operating room image & 18 & 82 & 100 \\
\hline Venoarteriel & 56 & 44 & 100 \\
\hline Venovenous & 48 & 52 & 100 \\
\hline Working principle & 56 & 44 & 100 \\
\hline Radiological examination & 23 & 77 & 100 \\
\hline Educative & 61 & 39 & 100 \\
\hline HD image & 64 & 36 & 100 \\
\hline ECMO: Extra-corporeal membrane oxygenation, HD: High-definition
\end{tabular}

Table 2. Video characteristics according to years and suitability assessment of materials groups (mean \pm standard deviation)

\begin{tabular}{|l|l|l|l|l|l|}
\hline Years & Follow-up & Like & Dislike & \multicolumn{1}{l}{ Comment } \\
\hline & Mean \pm SD & Mean \pm SD & Mean \pm SD & Mean \pm SD & Mean \pm SD \\
\hline 2010 and earlier $(n=4)$ & $22213.75 \pm 14961.07$ & $13.50 \pm 17.59$ & $2.25 \pm 3.86$ & $7.00 \pm 3.46$ & $2.50 \pm 1.91$ \\
\hline $2011-2015(n=36)$ & $26283.80 \pm 29114.14$ & $43.86 \pm 51.83$ & $3.19 \pm 3.70$ & $4.00 \pm 5.44$ & $8.02 \pm 9.01$ \\
\hline 2016 and later $(n=60)$ & $7675.70 \pm 12860.9$ & $28.33 \pm 46.71$ & $1.25 \pm 2.58$ & $1.21 \pm 2.16$ & $16.86 \pm 20.42$ \\
\hline p & $<\mathbf{0 . 0 0 1}$ & $\mathbf{0 . 0 6 3}$ & $\mathbf{0 . 0 0 5}$ & $<0.001$ & $\mathbf{0 . 0 1 8}$ \\
\hline SAM group & & & & $2.66 \pm 3.73$ \\
\hline Insufficient $(n=21)$ & $13055.23 \pm 15204.59$ & $23.42 \pm 30.30$ & $2.76 \pm 3.52$ & $6.80 \pm 9.81$ \\
\hline Sufficient $(n=49)$ & $8018.26 \pm 13344.69$ & $27.34 \pm 44.03$ & $1.02 \pm 2.05$ & $1.27 \pm 2.42$ & $18.67 \pm 20.72$ \\
\hline Superior $(n=30)$ & $27618.63 \pm 30901.27$ & $50.03 \pm 60.79$ & $3.03 \pm 3.99$ & $4.33 \pm 5.69$ & $8.43 \pm 11.96$ \\
\hline p & $\mathbf{0 . 0 0 2}$ & $\mathbf{0 . 6 3 2}$ & $\mathbf{0 . 0 4 0}$ & $\mathbf{0 . 0 1 5}$ & $\mathbf{0 . 0 2}$
\end{tabular}

SAM: Suitability assessment of material, SD: Standard deviation 
videos over the years $\left.{ }^{(1-3)}\right]$, (Table 4: number of videos per year).

The top three countries which had been the most ECMO video source were the USA, India and England. Only $64(64 \%)$ of the videos had high-definition (HD) image quality and this rate had significantly increased after 2015 $(\mathrm{p}<0.001)$. Number of views, likes, dislikes, comments, video lengths, overall and percentage of SAM scores were not statistically different in comparisons when the videos were classified according to country origin and presence/ absence of HD quality ( $>0.05)$.

\section{Discussion}

In this study, the relevance, content, and user engagement of the videos on YouTube about ECMO were evaluated.

Table 3. Comparison of the content of videos over the years

\begin{tabular}{|c|c|c|c|c|c|}
\hline Video content/years & & $\leq 2010, n(\%)$ & 2011-2015, n (\%) & $\geq 2016, n(\%)$ & $\mathbf{p}$ \\
\hline \multirow{2}{*}{ Animation } & + & $0(0 \%)$ & $4(11.1 \%)$ & $8(13.3 \%)$ & \multirow{2}{*}{0.714} \\
\hline & - & $4(100 \%)$ & $32(88.9 \%)$ & $52(86.7 \%)$ & \\
\hline \multirow{2}{*}{ Cannula procedure } & + & $0(0 \%)$ & $7(19.4 \%)$ & $10(16.7 \%)$ & \multirow{2}{*}{614} \\
\hline & - & $4(100 \%)$ & $29(80.6 \%)$ & $50(83.3 \%)$ & \\
\hline \multirow{2}{*}{ ECMO monitoring } & + & $0(0 \%)$ & $6(16.7 \%)$ & $26(43.3 \%)$ & \multirow{2}{*}{9} \\
\hline & - & $4(100 \%)$ & $30(83.3 \%)$ & $34(56.7 \%)$ & \\
\hline \multirow{2}{*}{ Decannulation } & + & $0(0 \%)$ & $2(5.6 \%)$ & $6(10 \%)$ & \multirow{2}{*}{617} \\
\hline & - & $4(100 \%)$ & $34(94.4 \%)$ & $54(90 \%)$ & \\
\hline \multirow{2}{*}{ Indication } & + & $0(0 \%)$ & $7(19.4 \%)$ & $30(50 \%)$ & \multirow{2}{*}{3} \\
\hline & - & $4(100 \%)$ & $29(80.6 \%)$ & $30(50 \%)$ & \\
\hline \multirow{2}{*}{ Complication } & + & $0(0 \%)$ & $8(22.2 \%)$ & $28(46.7 \%)$ & \multirow{2}{*}{17} \\
\hline & - & $4(100 \%)$ & $28(77.8 \%)$ & $32(53.3 \%)$ & \\
\hline \multirow{2}{*}{ Treatment management } & + & $0(0 \%)$ & $6(16.7 \%)$ & $27(45 \%)$ & \multirow{2}{*}{6} \\
\hline & - & $4(100 \%)$ & $30(83.3 \%)$ & $33(55 \%)$ & \\
\hline \multirow{2}{*}{ Complication prevention } & + & $0(0 \%)$ & $7(19.4 \%)$ & $24(40 \%)$ & \multirow{2}{*}{42} \\
\hline & - & $4(100 \%)$ & $29(80.6 \%)$ & $36(60 \%)$ & \\
\hline \multirow{2}{*}{ Operating room image } & + & $1(25 \%)$ & $6(16.7 \%)$ & $11(18.3 \%)$ & \multirow{2}{*}{914} \\
\hline & - & $3(75 \%)$ & $30(83.3 \%)$ & $49(81.7 \%)$ & \\
\hline \multirow{2}{*}{ Venoarteriel } & + & $0(0 \%)$ & $15(41.7 \%)$ & 41 (68.3\%) & \multirow{2}{*}{3} \\
\hline & - & $4(100 \%)$ & $21(58.3 \%)$ & $19(31.7 \%)$ & \\
\hline \multirow{2}{*}{ Venovenous } & + & $0(0 \%)$ & $13(36.1 \%)$ & $35(58.3 \%)$ & \multirow{2}{*}{16} \\
\hline & - & $4(100 \%)$ & $23(63.9 \%)$ & $25(41.7 \%)$ & \\
\hline \multirow{2}{*}{ Working principle } & + & $1(25 \%)$ & $14(38.9 \%)$ & $41(68.3 \%)$ & \multirow{2}{*}{8} \\
\hline & - & $3(75 \%)$ & $22(61.1 \%)$ & $19(31.7 \%)$ & \\
\hline \multirow{2}{*}{ Radiological examination } & + & $0(0 \%)$ & $5(13.9 \%)$ & $18(30 \%)$ & \multirow{2}{*}{103} \\
\hline & - & $4(100 \%)$ & $31(86.1 \%)$ & $42(70 \%)$ & \\
\hline \multirow{2}{*}{ Educative } & + & $0(0 \%)$ & $17(47.2 \%)$ & $44(73.3 \%)$ & \multirow{2}{*}{2} \\
\hline & + & $4(100 \%)$ & $19(52.8 \%)$ & $16(26.7 \%)$ & \\
\hline \multirow{2}{*}{ HD image } & + & $1(25 \%)$ & $14(38.9 \%)$ & $49(81.7 \%)$ & 5 \\
\hline & - & $3(75 \%)$ & $22(61.1 \%)$ & $11(18.3 \%)$ & $>0.00$ \\
\hline & Insufficient & $3(75 \%)$ & $8(22.2 \%)$ & $10(16.7 \%)$ & \\
\hline SAM & Sufficient & $0(0 \%)$ & $12(33.3 \%)$ & $37(61.7 \%)$ & 3 \\
\hline & Superior & $1(25 \%)$ & $16(44.4 \%)$ & $13(21.7 \%)$ & \\
\hline
\end{tabular}

ECMO: Extra-corporeal membrane oxygenation, HD: High-definition, SAM: Suitability assessment of material 
Table 4. Number of videos per year

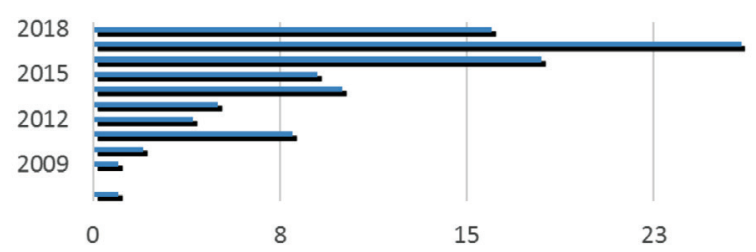

The number, content, diversity and duration of videos regarding ECMO which were uploaded YouTube increased significantly after 2016, however, there was no significant relationship between history and SAM score, however, there was no significant relationship between loading date and SAM score. Moreover, there is no significant correlation between the duration of video and SAM score. On the other hand, there was a positively weak correlation between the scores of the videos and the number of views.

While YouTube's content creators have designed it for entertainment rather than education, users share a large number of videos about medical issues that are related to themselves or for medical educational purposes. Without a standardized peer review process or a method where reliable sources can be identified, video searches on YouTube result in a vague mix of video and entertainmentoriented videos. The aim of this study was to evaluate the medical benefits of YouTube by evaluating ECMO videos and presenting them to the literature. We are opinion that such findings may contribute to the visual learning curve by examining what applications are shown about ECMO procedures on YouTube.

Nowadays, educational videos are now being frequently uploaded to the internet via social media sources in the cardiovascular field. When current articles reviewed in the literature concerning this issue, they emphasize that such videos benefit patient management and decision of methods to apply. For instance, Pitcher et al. reviewed common femoral artery interventions in YouTube and other online resources ${ }^{(3)}$. Although used by medical students, vascular experts are not sufficiently standardized to a basic vascular procedure for access to the femoral artery. However, there is no consensus about femoral interventions or established a certain vascular procedure.

The researchers who are well-known vascular specialists, emphasized that, vascular surgeons should improve the visibility of educational videos in online learning resources. Pandey et al. analyzed the results of Google search, YouTube videos, Twitter messages about left ventricular assist device-heart transplantation then they determined that numbers of studies, searches and upload have been significantly increased during last 7 years on the social media ${ }^{(4)}$. Similarly in our study, the increase in the number of videos in 2016 and beyond is remarkable. Compatible with this, an increase in the number of videos in 2016 and beyond was observed in our study as well.

Chen et al. implied the importance of YouTube videos in the education process in their study ${ }^{(5)}$. Furthermore, they stated that uploading more videos concerning heart transplantation with a HD would be better for this purpose. In our study, it was determined that the number of HD videos increased after 2015 in parallel with technological advances.

However, the characteristics of the video, such as number of view, like, dislike, comment, duration, SAM total score and percentages of SAM are not different in comparisons by being videos HD or not. Therefore, these outcomes are interpreted in favor of the quality of the image, content and audiences' responses are not increasing in parallel.

Agrawal et al. have addressed the need for care of in children with congenital coronary anomalies by conducting social media search ${ }^{(6)}$. On the other hand, Panhuyzen-Goedkoop et al. have evaluated the YouTube videos including sudden athlete deaths in order to evaluate the relation between facial expressions and type of syncope ${ }^{(7)}$. They pointed out the deficiencies in CPR onset and highlighted the waste of valuable time since the beginning of syncope.

Bademci et al. evaluated the YouTube videos regarding deep venous thrombus ${ }^{(8)}$. They determined that the videos which were uploaded by clinicians were more educational 
thus they suggested that more treatment-diagnostic based videos with animation should be loaded. Moreover, Gunes et al. remarked the importance of public information about varicose veins after evaluating social media videos considering the prevalence of disease ${ }^{(9)}$.

Azer et al. reported the lack of sufficient number of original educational video about the cardiovascular and respiratory system after they reviewed 1920 physical examination and training videos on YouTube ${ }^{(10)}$. Similarly, Desai et al. analyzed a total of 607 medical content videos on YouTube ${ }^{(11)}$. In this study, all videos were evaluated in terms of intelligibility using SAM and user participation measurements were obtained for each video. The researchers found that only $27 \%$ of the videos they analyzed had superior SAM score. This rate was $30 \%$ in our study.

Although the researchers emphasized that the videos with high SAM scores were more educational, they stated that they could not find a significant relationship between high SAM scores and user-based variables such as numbers of view, like, and comment. As a result, they declared that the concept of "content is king" is yet unclear whether it applies to medical videos uploaded by reliable sources for patient education on YouTube ${ }^{(11)}$. Compatible with this, we used the SAM score and determined that there was a positively weak correlation between the scores of the videos and the number of views. However, a significant correlation between SAM score and user participation parameters, numbers of like, dislike, comment was not determined in our study as well. Interestingly, we found that when videos classified in account of SAM score as insufficient, sufficient and superior, sufficient ones had less view number than insufficient ones. Nonetheless, both sufficient and insufficient groups had similar number of like. This evidence supports that content of video is not always parallel to user engagement assessments therefore the underlying reasons need to be investigated by further studies.

Chen et al. revealed a positive correlation between use of ECMO and the popular ECMO news on social media ${ }^{(12)}$. As a consequence, they concluded that social media is supportive for public knowledge about ECMO.

Over the last decade, social media has emerged as a new platform for information exchange and interpersonal communication for health professionals ${ }^{(13)}$. This progress in technology should be used to achieve better clinical results and to share information. However, the basic behaviors of health professionals and the increase of productivity of social media and their ethical use for a sustainable health system remain unclear. Inadequate information quality, patient confidentiality and legal issues are risks and challenges that can affect the effective and useful integration of online platforms. In this respect, our study aimed to clarify the factors related to the frequency of social media usage and user participation on YouTube videos regarding ECMO in the discipline of cardiovascular surgery. Thus we targeted to enhance the communication and collaboration among the health professionals. Consequently, such findings may benefit to get a better knowledge about the behavior of health professionals in their daily practices. To summarize, YouTube is being increasingly used as a platform for to share health information ${ }^{(14)}$.

Our research shows important results regarding the adoption of YouTube in healthcare practice. Based on the findings of this study, we determined that the use of YouTube video eases the information exchange especially among young people and increases the share of medical experiences that support ECMO's learning and development. Moreover, social media platforms contribute to develop a higher level of communication among practitioners and increase daily productivity. In this respect, health services can benefit from interactive platforms. We believe that YouTube video sharing and their awareness are useful in terms of process quality for to develop a vital application such as ECMO. The advancement of online technologies will promote practitioners in collecting, managing and interacting for better quality of service. YouTube increases the exchange of information between healthcare professionals about the optimal decision making process for ECMO initiation. 
YouTube's viral marketing potential may to change patients' beliefs about controversial medical issues ${ }^{(15)}$. A nonexpert user is more likely to find such content, and getting related videos depends on the search term used. In addition, healthcare seekers seem to be affected by the language styles of providers depending on web-based communication content ${ }^{(16)}$. YouTube is being used as a tool to promote nonscientific treatments and medicines that have not yet been approved by appropriate institutions ${ }^{(17)}$. The importance of video sharing, which has reached more than $90 \%$ of global internet traffic by 2020, is supported by a few estimates.

In our study, it has been found that the number, content, diversity of videos about ECMO have increased significantly in recent years. However, there was no significant relationship between upload date and content scores besides there was a positively weak correlation between the content score and the number of views.

In this respect, considering that the discussions in the future may be due to insufficient information quality, patient confidentiality and legal issues, we are in opinion that the necessity of double blind peer review process for social media shares will be one of the most important point to consider.

\section{Ethics}

Ethics Committee Approval: Our study is based on a web research therefore ethical approval did not obtained.

Informed Consent: This study does not include any patient, for this reason there was no need to obtain an informed consent form.

Peer-review: Externally and internally peer-reviewed.

\section{Authorship Contributions}

Surgical and Medical Practices: T.G., Ç.B., Concept: S.B., G.A., Design: V.H., S.B., Data Collection or Processing: T.G., Ç.B., Analysis or Interpretation: S.B., G.A., Literature Search: V.H., S.B., Writing: T.G.

Conflict of Interest: No conflict of interest was declared by the authors.

Financial Disclosure: The authors declared that this study received no financial support.

\section{References}

1. Deal SB, Alseidi AA. Concerns of Quality and Safety in Public Domain Surgical Education Videos: An Assessment of the Critical View of Safety in Frequently Used Laparoscopic Cholecystectomy Videos. J Am Coll Surg 2017;225:725-30.

2. Raikos A, Waidyasekara P. How useful is YouTube in learning heart anatomy? Anat Sci Educ 2014;7:12-8.

3. Pitcher GS, Newton DH, Amendola MF. Common Femoral Artery Access on YouTube: What Practices are Being Shown and Who is Delivering the Message? J Surg Educ 2017;74:455-8.

4. Pandey A, Abdullah K, Drazner MH. Impact of Vice President Cheney on public interest in left ventricular assist devices and heart transplantation. Am J Cardiol 2014;113:1529-31.

5. Chen HM, Hu ZK, Zheng XL et al. Effectiveness of YouTube as a Source of Medical Information on Heart Transplantation. Interact J Med Res 2013;2:e28.

6. Agrawal H, Wright OK, Carberry KE, et al. Family perception of unmet support needs following a diagnosis of congenital coronary anomaly in children: Results of a survey. Congenit Heart Dis 2017;12:721-5.

7. Panhuyzen-Goedkoop NM, Wellens HJ, Piek JJ. Early recognition of sudden cardiac arrest in athletes during sports activity. Neth Heart $\mathbf{J}$ 2018;26:21-5

8. Bademci MS, Yazman S, Gunes T, et al. YouTube as a potential source of information on deep venous thrombosis. Phlebology 2017;32:543-7.

9. Gunes T, Serinken M, Alur I, et al. YouTube as a source of information on varicose veins. Phlebology 2016;31:501-5.

10. Azer SA, AlGrain HA, AlKhelaif RA, et al. Evaluation of the educational value of YouTube videos about physical examination of the cardiovascular and respiratory systems. J Med Internet Res 2013;15:e241.

11. Desai T, Shariff A, Dhingra V, et al. Is content really king? An objective analysis of the public's response to medical videos on YouTube. PLoS One 2013;8:e82469.

12. Chen YY, Chen L, Huang TS, et al. Significant social events and increasing use of life-sustaining treatment: trend analysis using extracorporeal membrane oxygenation as an example. BMC Med Ethics 2014;15:21.

13. Hazzam J, Lahrech A. Health Care Professionals' Social Media Behavior and the Underlying Factors of Social Media Adoption and Use: Quantitative Study. J Med Internet Res 2018;20:e12035.

14. Madathil KC, Rivera-Rodriguez AJ, Greenstein JS, Gramopadhye AK, et al. Healthcare information on YouTube: A systematic review. Health Informatics J 2015;21:173-94.

15. Godwin HT, Khan M, Yellowlees P. The Educational Potential of YouTube Acad Psychiatry 2017;41:823-7.

16. Zimmermann M, Jucks R. Investigating the Role of Communication for Information Seekers' Trust-Related Evaluations of Health Videos on the Web: Content Analysis, Survey Data, and Experiment. Interact J Med Res 2018;7:e10282.

17. Hucíková A, Babic A. Medical Informatics Idle YouTube Potential. Stud Health Technol Inform 2017;238:132-5. 